\title{
Squeezing Force of the Magnetorheological Fluid Isolating Damper for Centrifugal Fan in Nuclear Power Plant
}

\author{
Jin Huang, ${ }^{1,2}$ Ping Wang, ${ }^{1}$ and Guochao Wang ${ }^{1}$ \\ ${ }^{1}$ Chongqing Institute of Automobile, Chongqing University of Technology, Chongqing 400054, China \\ ${ }^{2}$ The Key Laboratory of Manufacture and Test Techniques for Automobile Parts, Chongqing University of Technology, \\ Chongqing 400054, China
}

Correspondence should be addressed to Jin Huang, jhuangcq@163.com

Received 15 June 2012; Revised 3 September 2012; Accepted 8 September 2012

Academic Editor: Yan Yang

Copyright (c) 2012 Jin Huang et al. This is an open access article distributed under the Creative Commons Attribution License, which permits unrestricted use, distribution, and reproduction in any medium, provided the original work is properly cited.

\begin{abstract}
Magnetorheological (MR) disk-type isolating dampers are the semi-active control devices that use MR fluids to produce controllable squeezing force. In this paper, the analytical endeavor into the fluid dynamic modeling of an MR isolating damper is reported. The velocity and pressure distribution of an MR fluid operating in an axisymmetric squeeze model are analytically solved using a biviscosity constitutive model. Analytical solutions for the flow behavior of MR fluid flowing through the parallel channel are obtained. The equation for the squeezing force is derived to provide the theoretical foundation for the design of the isolating damper. The result shows that with the increase of the applied magnetic field strength, the squeezing force is increased.
\end{abstract}

\section{Introduction}

With the continuous construction of nuclear power plants in China, more and more attentions are paid to safety and reliability of the auxiliary equipments in nuclear power plant. There is a pressing need to researching earthquake resistance of auxiliary equipment in the nuclear power plant. The centrifugal fan in the nuclear power plant must have good aseismatic performance that is the important condition for the safe operation of nuclear power plants in the earthquake. However, there have been few reports on vibration insulator for centrifugal fan $[1,2]$. Earthquake response attenuation of centrifugal fans can be improved by connecting them with isolator. In this paper, we propose an isolating damper based on magnetorheological (MR) for centrifugal fan.

MR fluids are materials of micron-sized and magnetized particles in a carrier fluid. In the absence of an applied magnetic field, MR fluids flow freely. The fluids exhibit Newtonian-like behavior. Upon application of a magnetic field, these fluids exhibit viscoplastic behavior with yield strength [3]. Altering the strength of an applied magnetic field will precisely control the shear yield stress of the fluid. Based on the mechanical characteristics, the fluids can be used in the magnetically controlled devices such as brakes $[4]$ and dampers $[5,6]$.

The MR isolating damper is one such device that provides controllable squeezing force. Altering the strength of an applied magnetic field will change the squeezing force of the MR isolating damper [7]. The research of MR dampers and their applications have been done in many different ways. In the field of the flow model description for MR damper, Boelter and Janocha [8] analyzed the working mode (shear mode, flow mode, squeeze mode) in MR damper. Mcmanus et al. [9] studied the squeeze flow mode in accompany with shear in MR damper, analyzed the damper force-velocity characteristics under different values of magnetic field strength. For the research of application in building structures, Motra et al. [10] researched the response attenuation of seismically excited adjacent buildings connected by a MR damper. P. Y. Lin and T. K. Lin [11] introduced a bridge isolation system that combines the rolling pendulum system (RPS) and the MR damper. Dragasius et al. [12] researched the resistance force generated by linear hydrocylinder-type magnetorheological fluid (MRF) damper acting in different regimes. It was defined that the force increases practically linearly when increasing strength of the magnetic field and 


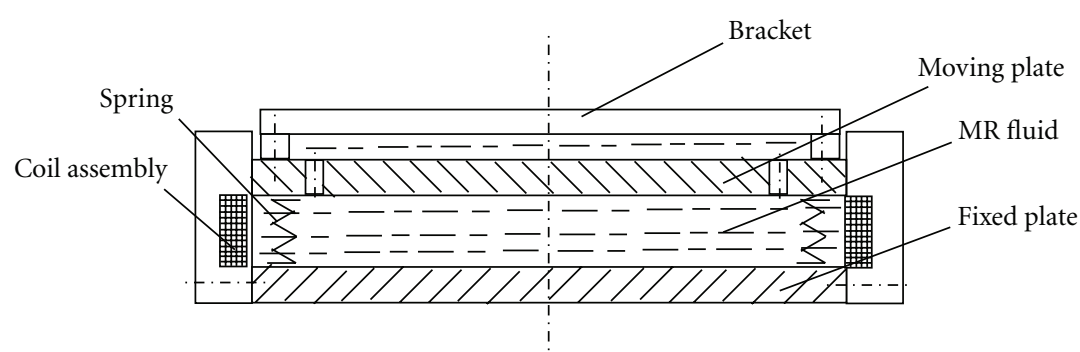

FIGURE 1: Operational principle of a circular plate MR isolating damper.

when increasing piston speed. Erkus and Johnson [13] investigated the dissipativity and performance characteristics of the semiactive control of the base-isolated benchmark structure with MR dampers. Rajamani and Larparisudthi [14] proposed a vibration control model for MR isolating damper.

In this paper, biviscosity model is used to describe the constitutive characteristics of MR fluids subject to an applied magnetic field. The operational principle of the MR isolating damper is introduced. Analytical solutions for the axisymmetric squeeze flow behavior of a biviscosity fluid are obtained. The velocity equation and the location of the unyield flow region are obtained. The expression for the squeezing force is derived to provide the theoretical foundation for the design of the isolating damper. The result shows that with the increase of the applied magnetic field strength, the dynamic yield stress of the MR fluid goes up rapidly, and the squeezing force is increased.

\section{Operational Principle}

The schematic configuration of the proposed circular plate MR isolating damper is shown in Figure 1. The MR isolating damper consists of an MR fluid, moving plate, working gap, electromagnet coil, flux guide, and housing. The MR fluid fills the working gap between the moving plate and housing. The electromagnet coil in the housing provides the magnetic field in the working gap. During relative vertical motion between the moving plate and housing, MR fluid is squeezed in the working gap. Thus, the pressure drop due to flow resistance of MR fluid in the working gap is induced. The MR isolating damper produces a controllable squeezing force due to the yield stress of the MR fluid, if a certain level of magnetic field is applied through the working gap.

\section{Modeling and Analysis}

3.1. Analysis of Flow Velocity in Working Gap. Figure 2 shows a schematic of the axisymmetric squeeze flow model. The gap between the disks is filled with the MR fluid. The bottom disk is fixed, while the top disk approaches the bottom disk with a constant velocity $U$. Gravity is neglected, so that the $z=h / 2$ plane is a plane of symmetry for the flow. The force acting on bottom disk is

$$
F=\int_{0}^{R} 2 \pi r p d r
$$

where, $p$ is the pressure, $R$ is radius of the disks.

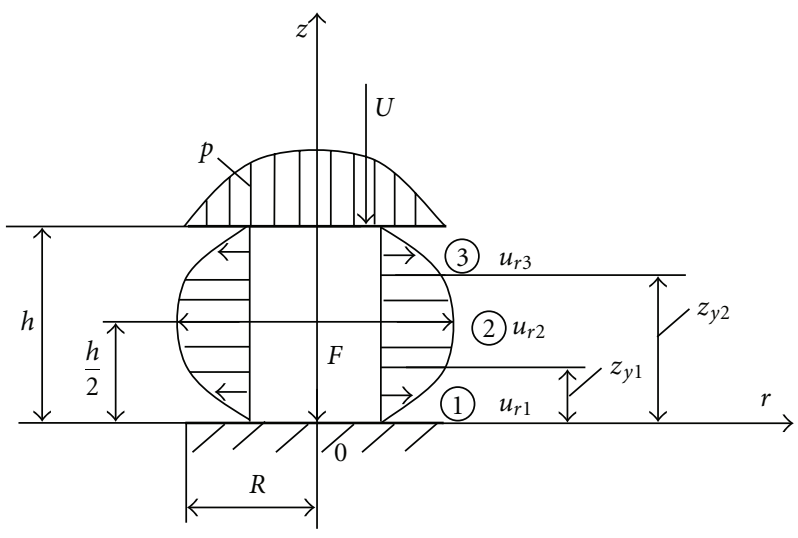

FIgURE 2: Squeeze model of MR fluid between two parallel disks.

To circumvent the "squeeze-flow paradox" of the Bingham model [15] and to facilitate an analytical analysis, the biviscosity constitutive model is employed in this study to describe the behavior of the MR fluid, see in Figure 3. It is known that the biviscosity model can provide very useful and convenient ways for the calculation of materials' behavior with yield, particularly from an analytical perspective.

The constitutive equation of the biviscosity model can be represented by the following two expressions [16]:

$$
\begin{array}{ll}
\tau=\tau_{0}+\eta \frac{d u_{r}}{d z}, & |\tau| \geq \tau_{y}(H), \\
\tau=\eta(H) \frac{d u_{r}}{d z}, & |\tau| \leq \tau_{y}(H),
\end{array}
$$

where $\tau$ is the shear stress, $\tau_{y}(H)$ is the dynamic yield stress developed in response to an applied magnetic field $H, \tau_{0}$ is the intercept stress, $\eta$ is the viscosity of the fluid when stress is higher than the dynamic yield stress, $\eta(H)$ is the viscosity coefficient subjected to a stress lower than the dynamic yield stress, it's a function of the magnetic field strength. Viscosity ratio $\varepsilon=\eta / \eta(H)$ is an important parameter for the biviscosity constitutive model, $\tau_{0}=(1-\varepsilon) \tau_{y}(H)$. Note that when $\varepsilon \rightarrow 0,(2 \mathrm{a})$ and (2b) are the Bingham constitutive model, whereas when $\varepsilon=1$ they are the Newtonian constitutive model.

It is evident from the constitutive equation that the biviscosity constitutive model can be divided into two regions based on the dynamic yield stress. The MR fluid to be yielded 


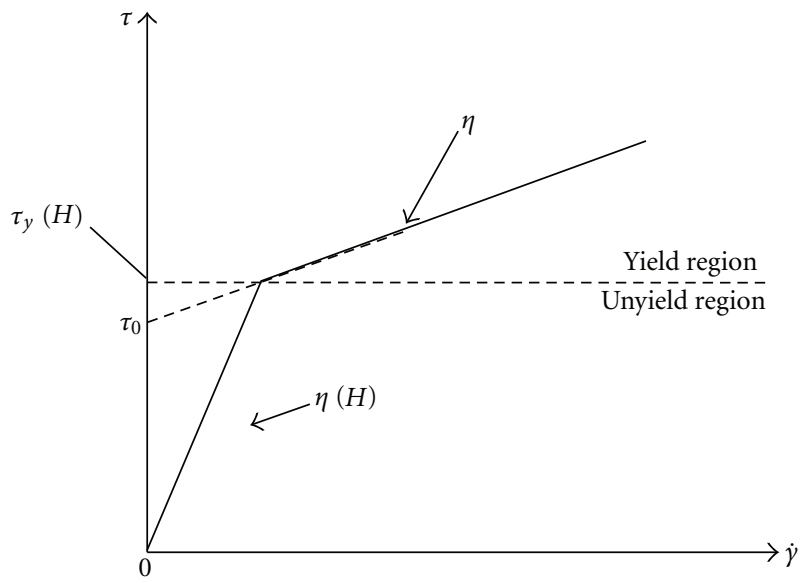

FIgURE 3: Biviscosity model of MR fluid.

when the magnitude of the fluid's internal stress is greater than the yield stress $\tau_{y}(H)$, the fluid exhibits typical Newtonian behavior. The MR fluid is unyield when the magnitude of the fluid's internal stress is smaller than $\tau_{y}(H)$, the fluid flows slowly with highly viscous.

For the disc-type squeeze flow model in Figure 2, assuming steady state condition $(\partial / \partial t=0)$, symmetry $(\partial / \partial \theta=0)$, no tangential and axial velocity $\left(u_{\theta}=u_{z}=0\right)$ but only radial, no body force, and no pressure gradient in thickness direction, the Navier-Stokes equation in the $r$ direction for the cylindrical coordinates $(r, \theta, z)$ is simplified to

$$
\eta \frac{d^{2} u_{r}}{d z^{2}}=-m
$$

where $u_{r}$ is the radial velocity, $m=-d p / d r, d p / d r$ is the pressure gradient in radial direction.

By integrating (3), the velocity profile can be easily obtained as

$$
u_{r}=-\frac{m}{2 \eta} z^{2}+A_{1} z+A_{2},
$$

where $A_{1}$ and $A_{2}$ symbolize the integral constants.

The MR fluid exhibits Newtonian behavior in the absence of an applied magnetic field, applying boundary conditions of $u_{r}=0$ at $z=0$ and $z=h$, the flow velocity $u_{r 0}$ can be obtained as follows:

$$
u_{r 0}=\frac{m}{2 \eta} z(h-z)
$$

According to the assumptions, the momentum equation in the $r$-direction is

$$
\frac{d \tau_{z r}}{d z}=-m
$$

By integrating (6) along the thickness direction and applying boundary condition of $\tau_{z r}=0$ at $z=h / 2$, the following equation for the shear stress can be obtained:

$$
\tau_{z r}=m\left(\frac{h}{2}-z\right) .
$$

Assume that $z_{y_{1}}$ and $z_{y_{2}}$ denote the position of the unyield region boundaries measured from the bottom wall, respectively, see Figure 2. The yield surface is the locus of points where the shear stress is equal to the yield stress $(|\tau|=$ $\tau_{y}(H)$ ), the location of the unyield region can be determined by satisfying the conditions such that

$$
\begin{aligned}
& z_{y_{1}}=\frac{h}{2}-\frac{\tau_{y}(H)}{m}, \\
& z_{y_{2}}=\frac{h}{2}+\frac{\tau_{y}(H)}{m} .
\end{aligned}
$$

As seen in Figure 2, the flow is composed of three regions: two yield regions $\left(0 \leq z \leq z_{y_{1}}, z_{y_{2}} \leq z \leq h\right)$ contacting the bottom and top walls and an unyield region $\left(z_{y_{1}} \leq z \leq z_{y_{2}}\right)$.

In yield region $0 \leq z \leq z_{y_{1}}$, the two boundary conditions are

$$
\begin{aligned}
u_{r}=0 & \text { at } z=0, \\
\frac{d u_{r}}{d z}=0 & \text { at } z=z_{y_{1}} .
\end{aligned}
$$

Using the boundary conditions (9a) and (9b), determining the integral constant in (4), the velocity profile in yield region $0 \leq z \leq z_{y_{1}}$ can be obtained as

$$
u_{r 1}=\frac{m}{2 \eta}\left(h z-z^{2}\right)-\frac{\tau_{y}(H)}{\eta} z, \quad 0 \leq z \leq z_{y_{1}} .
$$

In unyield region $z_{y_{1}} \leq z \leq z_{y_{2}}$, the two boundary conditions are

$$
\begin{array}{cc}
u_{r}=u_{r 1} \quad \text { at } z=z_{y_{1}}, \\
\frac{d u_{r}}{d z}=0 \quad \text { at } z=\frac{h}{2} .
\end{array}
$$

The velocity profile in unyield region $z_{y_{1}} \leq z \leq z_{y_{2}}$ can be obtained by using the boundary conditions (11a) and (11b) as

$$
\begin{array}{r}
u_{r 2}=\frac{m \varepsilon}{2 \eta}\left(h z-z^{2}\right)+m \frac{1-\varepsilon}{2 \eta}\left(h z_{y_{1}}-z_{y_{1}}^{2}\right)-\frac{\tau_{y}(H)}{\eta} z_{y_{1}}, \\
z_{y_{1}} \leq z \leq z_{y_{2}} .
\end{array}
$$

Similar to the previous analysis, by using the boundary conditions of $u_{r}=0$ at $z=h$ and $d u_{r} / d z=0$ at $z=z_{y_{2}}$, the velocity profile in yield region $z_{y_{2}} \leq z \leq h$ can be obtained as

$$
u_{r 3}=\frac{m}{2 \eta}\left(h z-z^{2}\right)-\frac{\tau_{y}(H)}{\eta}(h-z), \quad z_{y_{2}} \leq z \leq h .
$$

3.2. Pressure Distribution. The pressure gradient $d p / d r$ can be determined by using the law of conservation of mass

$$
\frac{D}{D t} \int_{V} \rho d V=0
$$


where, $\rho$ is the density of MR fluid, $V$ is the volume of MR fluid. Equation (14) indicates that the derivative of liquid mass is zero

$$
\int_{V} \frac{\partial \rho}{\partial t} d V+\int_{S} \rho u_{j} n_{j} d s=0
$$

where, $s$ is the integral area, $u_{j}$ and $n_{j}$ denote the velocity tensor and direction cosines.

When $z_{y_{1}}=h / 2-\tau_{y}(H) / m \leq 0$, the MR fluid flows slowly with highly viscous, the pressure gradient can be expressed as

$$
\frac{d p}{d r}=\frac{6 \eta(H) r}{h^{3}} U
$$

When $z_{y_{1}}=h / 2-\tau_{y}(H) / m>0$, the MR fluid flows with a floating core, submitting (10), (12), (13) into (15) is

$$
\pi r^{2} U+2 \pi r\left(\int_{0}^{z_{y_{1}}} u_{r 1} d z+\int_{z_{y_{1}}}^{z_{y_{2}}} u_{r 2} d z+\int_{z_{y_{2}}}^{h} u_{r 3} d z\right)=0,
$$

where

$$
\begin{aligned}
\int_{0}^{z_{y_{1}}} u_{r 1} d z= & \frac{m h}{4 \eta} z_{y_{1}}^{2}-\frac{m}{6 \eta} z_{y_{1}}^{3}-\frac{\tau_{y}(H)}{2 \eta} z_{y_{1}}^{2}, \\
\int_{z_{y_{1}}}^{z_{y_{2}}} u_{r 2} d z= & 2 \int_{z_{y_{1}}}^{h / 2} u_{r 2} d z \\
= & \frac{m h \varepsilon}{2 \eta}\left(\frac{h^{2}}{4}-z_{y_{1}}^{2}\right)-\frac{m \varepsilon}{3 \eta}\left(\frac{h^{3}}{8}-z_{y_{1}}^{3}\right) \\
& +\frac{1-\varepsilon}{\eta} m h z_{y_{1}}\left(\frac{h}{2}-z_{y_{1}}\right)-2 m z_{y_{1}}\left(\frac{h}{2}-z_{y_{1}}\right), \\
& -\frac{2 \tau_{y}(H) z_{y_{1}}}{\eta}\left(\frac{h}{2}-z_{y_{1}}\right), \\
\int_{z_{y_{2}}}^{h} u_{r 3} d z= & \int_{0}^{z_{y_{1}}} u_{r 1} d z .
\end{aligned}
$$

So

$h^{3} m^{3}+\left[6 U r \eta-(1-\varepsilon) 3 h^{2} \tau_{y}(H)\right] m^{2}+4(1-\varepsilon) \tau_{y}^{3}(H)=0$.

3.3. Squeezing Force. The force acting on the bottom disc can be obtained by integrating the pressure along the radial direction:

$$
F=\int d F=\int_{0}^{R} 2 \pi r p d r .
$$

The subsection integral is used to get the force as

$$
F=\left[\pi p r^{2}\right]_{0}^{R}-\int_{0}^{R} \pi r^{2} \frac{d p}{d r} d r .
$$

The boundary conditions of pressure are

$$
\frac{d p}{d r}=0 \quad \text { at } r=0, \quad p=0 \quad \text { at } r=R .
$$

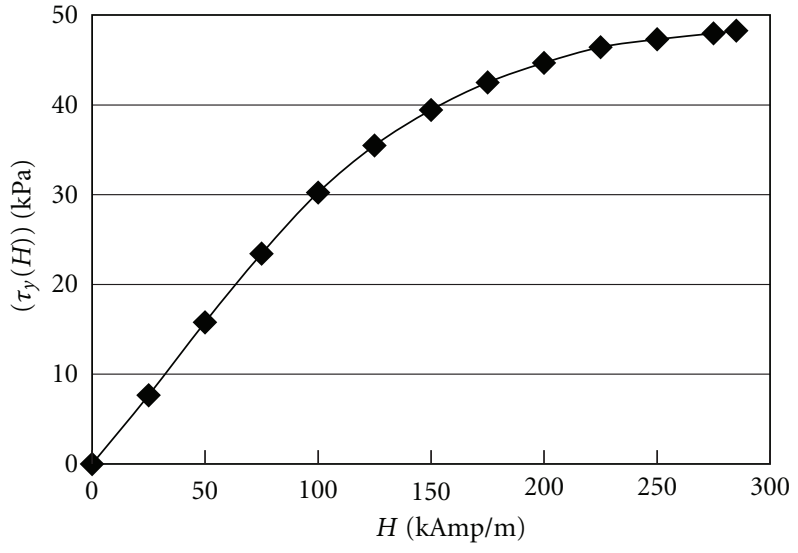

FIGURE 4: Yield strength versus magnetic field strength.

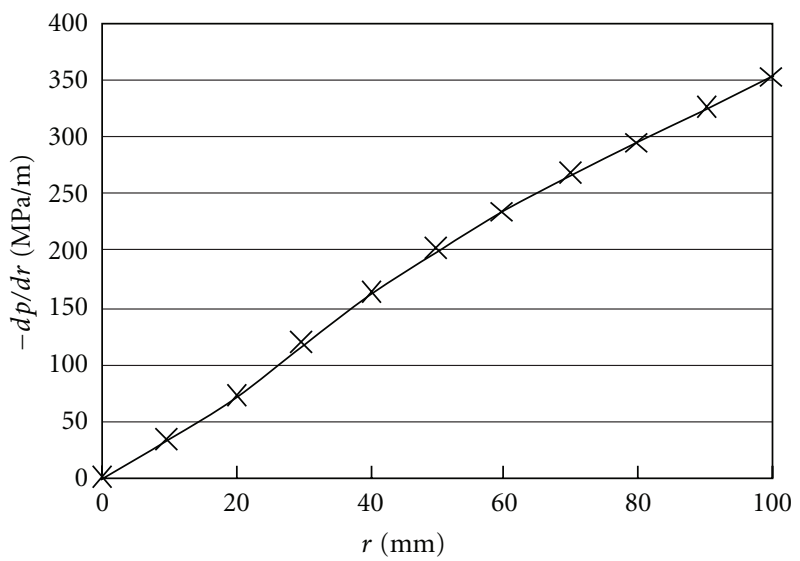

FIGURE 5: The pressure gradient versus radius.

Substituting (21) into (20), the force acting on the bottom disc can be obtained as

$$
F=-\pi \int_{0}^{R} r^{2} m d r
$$

\section{Results and Discussion}

A typical MR fluid is used in this paper. Figure 4 shows the yield strength of MR fluid under different magnetic field strength, measured by experiment. For the purpose of illustration, the following parameters are given: $h=1 \mathrm{~mm}$, $R=100 \mathrm{~mm}, U=5 \mathrm{~m} / \mathrm{s}, \eta=0.092 \mathrm{~Pa} \cdot \mathrm{s}$, and $\varepsilon=0.001$.

The relationship between the pressure gradient and the radius with apparent yield stress value of $30 \mathrm{kPa}$ is shown in Figure 5 under the application of magnetic field strength of $100 \mathrm{kAmp} / \mathrm{m}$ by solving (16) and (19). The absolute value of the pressure gradient increases with the radius in linear, approximately.

When the strength of magnetic field is $100 \mathrm{kAmp} / \mathrm{m}$, the yield surface determined by $(8 \mathrm{a})$ and ( $8 \mathrm{~b})$ with apparent yield stress value of $30 \mathrm{kPa}$ along the radial direction is shown in Figure 6. When $r \leq 17.7 \mathrm{~mm}$, the MR fluid is unyield totally, 


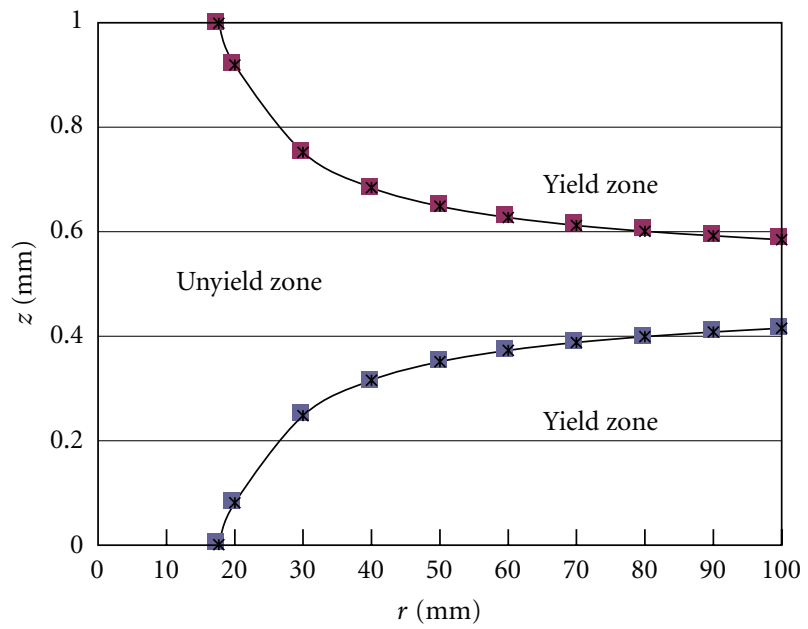

Figure 6: The yield surface along the radial direction.

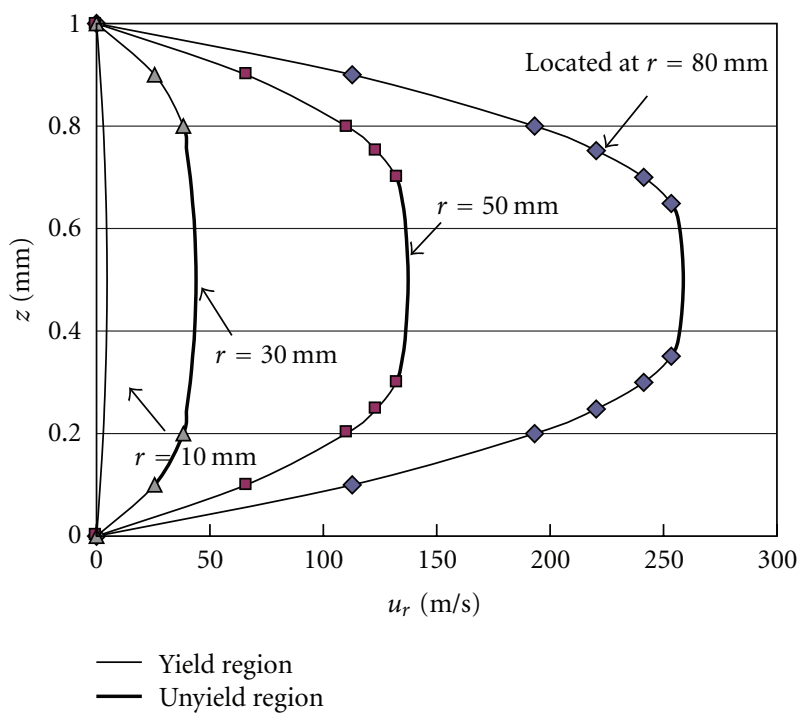

FIGURE 7: Velocity profiles for different location of radius.

flowing slowly with highly viscous. When $r>17.7 \mathrm{~mm}$, the MR fluid is yielded partially. The location of yield surface tends to move toward the symmetry plane of working gap as the radius increases.

The velocity profiles can be obtained by (10), (12), and (13) with apparent yield stress value of $30 \mathrm{kPa}$, shown in Figure 7. The velocity profiles for the squeeze flow of MR fluid appear to be parabolic curves with respect to $z$, and their curvature tends to decrease with radius, the unyield region decreases accordingly.

The squeezing force versus magnetic field strength is shown in Figure 8. In the absence of an applied magnetic field, the force is $216 \mathrm{kN}$. The forces are $250 \mathrm{kN}, 278 \mathrm{kN}$, and $302 \mathrm{kN}$ at the strength of magnetic field of $50 \mathrm{kAmp} / \mathrm{m}$, $100 \mathrm{kAmp} / \mathrm{m}$, and $200 \mathrm{kAmp} / \mathrm{m}$, respectively. The results indicate that with the increase of the velocity and the applied magnetic field, the squeezing force is increased.

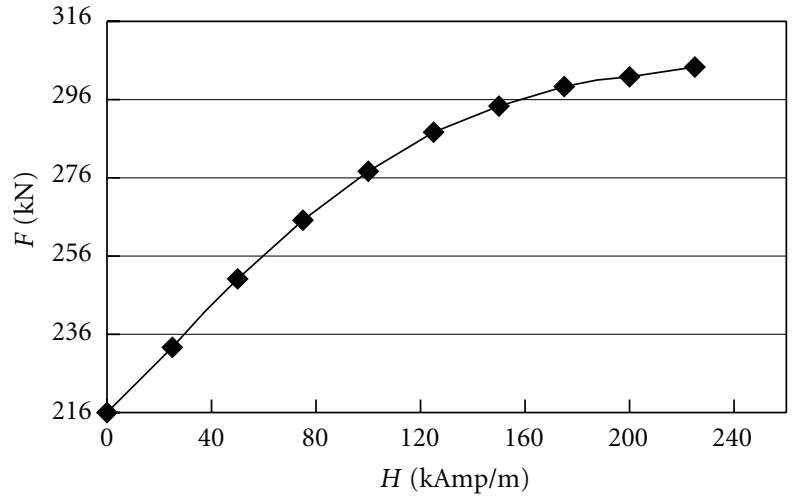

FIGURE 8: Squeezing force versus magnetic field strength.

\section{Conclusions}

The flow behaviors of MR fluid in circular plate MR isolating damper are investigated theoretically in this paper. The equations for the velocity and the squeezing force are derived to provide the theoretical foundation for the design of the MR isolating damper. The unyield region of MR fluid tends to move toward the symmetry plane of working gap as the radius increases. With the increase of the applied magnetic field strength, the squeezing force increased.

\section{Acknowledgments}

This work is supported by Project 51175532 by the National Natural Science Foundation of China and key Project 2011BA4028 by Natural Science Foundation Project of CQ CSTC.

\section{References}

[1] B. L. Wu, "Simply design for absorbing base of centrifugal fan," Compressor Blower \& Fan Technology, no. 6, pp. 46-47, 2000 (Chinese).

[2] H. Higashimori, K. Kuma, M. Goto, K. Kimura, and M. Koga, "Centrifugal fan has two sets of inlet damper blades, with one set controlled to form opening different from that formed by other set," US patent, JP2005188390-A, 2005.

[3] J. Huang, J. Q. Zhang, and J. N. Liu, "Effect of magnetic field on properties of MR fluids," International Journal of Modern Physics B, vol. 19, no. 1-3, pp. 597-601, 2005.

[4] J. Huang, J. Q. Zhang, Y. Yang, and Y. Q. Wei, "Analysis and design of a cylindrical magneto-rheological fluid brake," Journal of Materials Processing Technology, vol. 129, no. 1-3, pp. 559-562, 2002.

[5] K. K. Ahn, D. Q. Truong, and M. A. Islam, "Modeling of a magneto-rheological (MR) fluid damper using a self tuning fuzzy mechanism," Journal of Mechanical Science and Technology, vol. 23, no. 5, pp. 1485-1499, 2009.

[6] J. Huang, J. He, and G. Lu, "Analysis and design of magnetorheological damper," Advanced Materials Research, vol. 148149, pp. 882-886, 2011.

[7] G. Yang, B. F. Spencer, J. D. Carlson, and M. K. Sain, "Largescale MR fluid dampers: modeling and dynamic performance 
considerations," Engineering Structures, vol. 24, no. 3, pp. 309323, 2002.

[8] R. Boelter and H. Janocha, "Design rules for MR fluid actuators in different working modes," in Smart Structures and Materials 1997: Passive Damping and Isolation, vol. 3045 of Proceedings of SPIE, pp. 148-159, March 1997.

[9] S. J. McManus, K. A. St. Clair K.A., P. É. Boileau, J. Boutin, and S. Rakheja, "Evaluation of vibration and shock attenuation performance of a suspension seat with a semi-active magnetorheological fluid damper," Journal of Sound and Vibration, vol. 253, no. 1, pp. 313-327, 2002.

[10] G. B. Motra, W. Mallik, and N. K. Chandiramani, "Semi-active vibration control of connected buildings using magnetorheological dampers," Journal of Intelligent Material Systems and Structures, vol. 22, no. 16, pp. 1811-1827, 2011.

[11] P. Y. Lin and T. K. Lin, "Control of seismically isolated bridges by magnetorheological dampers and a rolling pendulum system," Structural Control and Health Monitoring, vol. 19, no. 2, pp. 278-294, 2012.

[12] E. Dragasius, V. Grigas, D. Mazeika, and A. Sulginas, "Evaluation of the resistance force of magnetorheological fluid damper," Journal of Vibroengineering, vol. 14, no. 1, pp. 1-6, 2012.

[13] B. Erkus and E. A. Johnson, "Dissipativity analysis of the base isolated benchmark structure with magnetorheological fluid dampers," Smart Materials \& Structures, vol. 20, no. 10, Article ID 105001, 2011.

[14] R. Rajamani and S. Larparisudthi, "On invariant points and their influence on active vibration isolation," Mechatronics, vol. 14, no. 2, pp. 175-198, 2004.

[15] S. D. R. Wilson, "Squeezing flow of a Bingham material," Journal of Non-Newtonian Fluid Mechanics, vol. 47, pp. 211219, 1993.

[16] K. Zhu, R. Ge, and B. Xi, "Squeezing flow of electrorheological fluid between two circular plates," Journal of Tsinghua University, vol. 39, no. 8, pp. 80-83, 1999 (Chinese). 

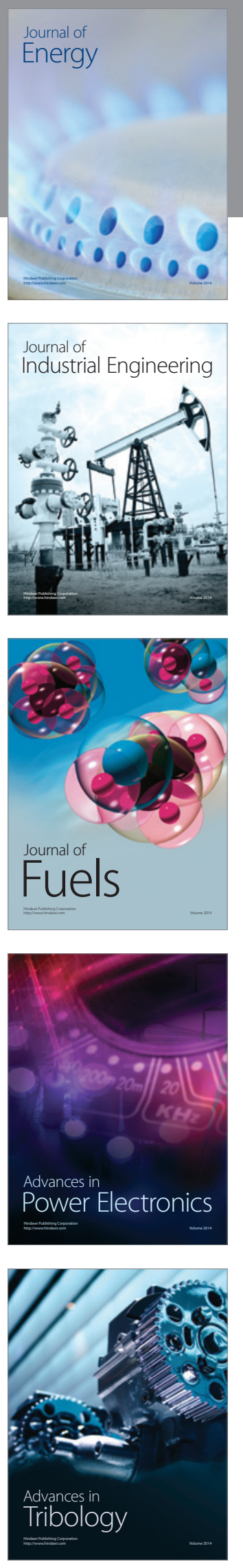
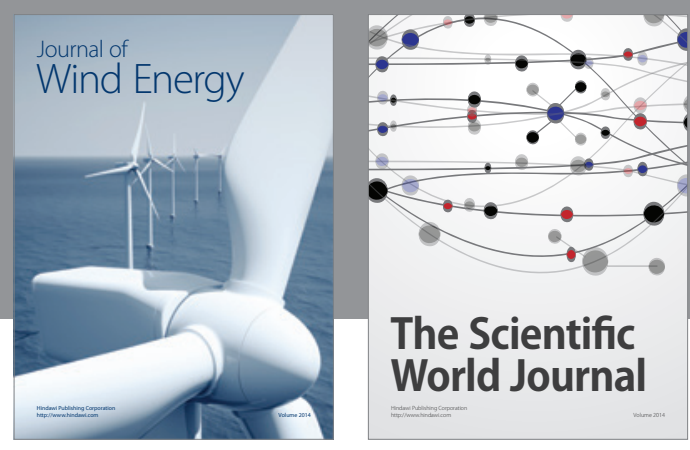

The Scientific World Journal

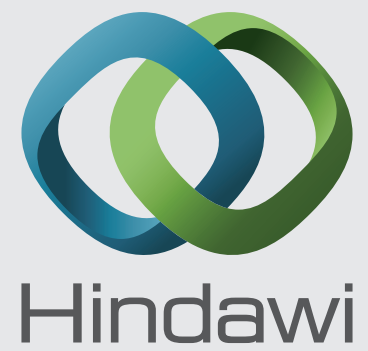

Submit your manuscripts at http://www.hindawi.com
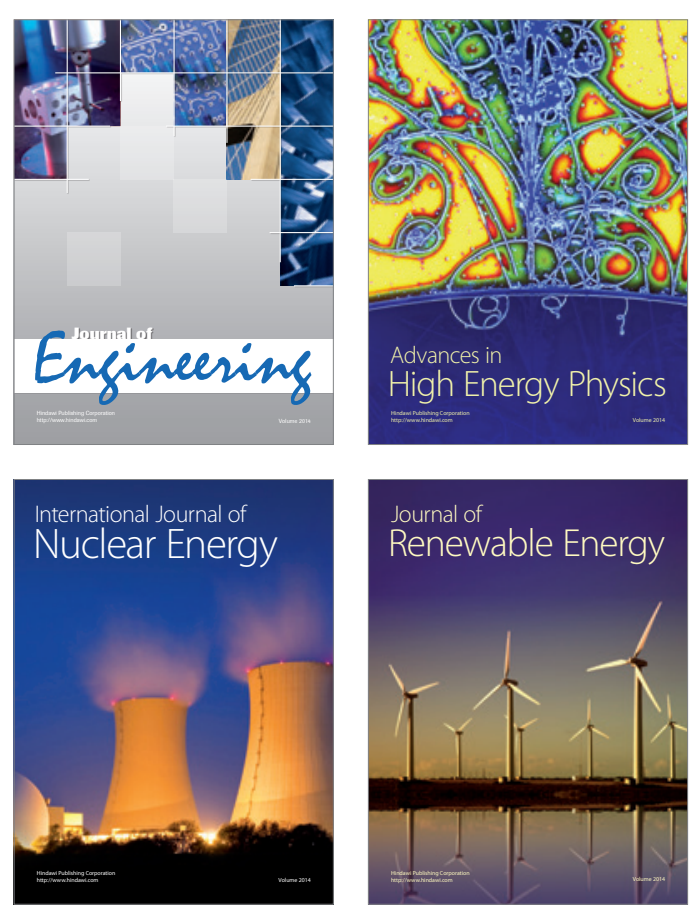

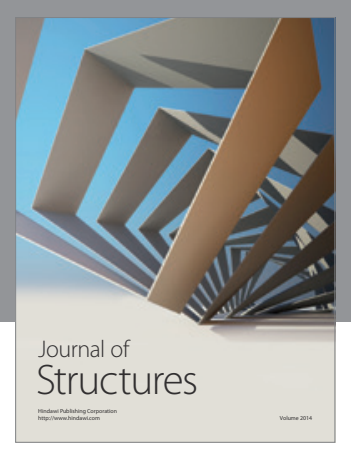

Rotating
Mechinery
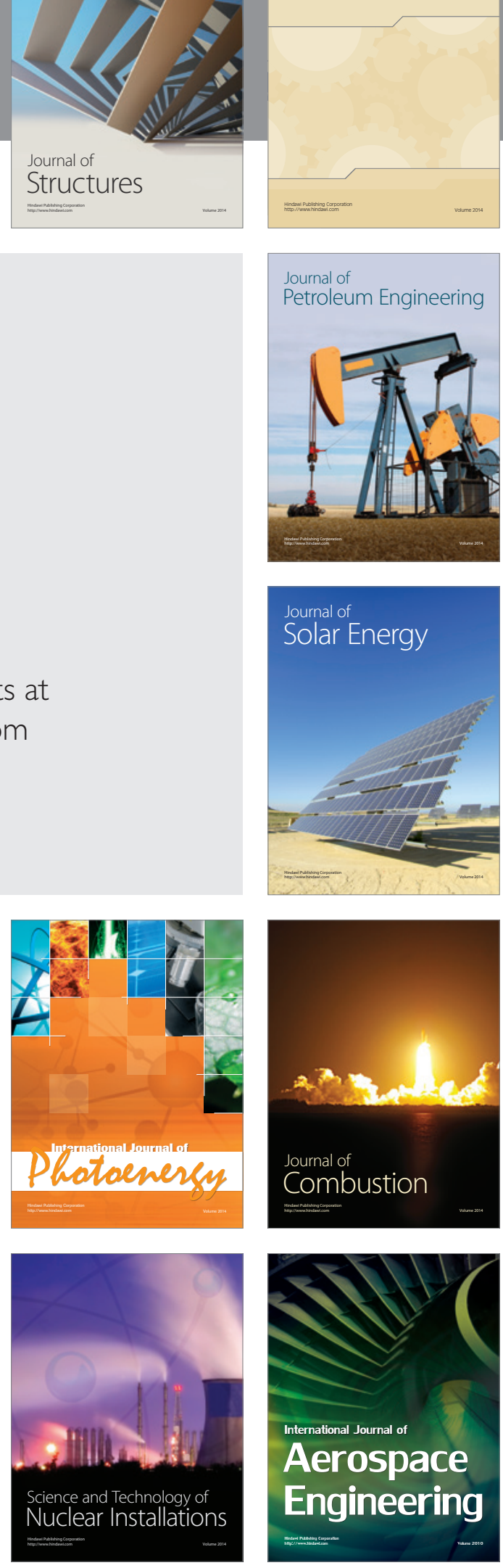